\title{
Modélisation de la climatologie nivale des Alpes françaises et application aux études d'impact des changements du climat
}

\author{
E. Martin, E. Brun et Y. Durand \\ Météo-France, Centre national de recherches météorologiques \\ Centre d'études de la neige, Saint Martin d'Hères
}

\begin{abstract}
L'existence d'un manteau neigeux saisonnier est une des caractéristiques importantes du climat des régions montagneuses des zones tempérées. Mais l'observation de l'enneigement dans les Alpes montre que celui-ci est extrêmement variable, à la fois dans l'espace et dans le temps. Cette variabilité est liée, par le biais de nombreux processus physiques, à celle des paramètres météorologiques. Compte tenu de cette relation, il faut s'attendre à ce qu'une modification du climat de l'Europe ait un impact important sur les conditions d'enneigement dans les Alpes, avec des conséquences importantes sur l'hydrologie et l'économie de toute la zone. Actuellement, les modèles de circulation générale (MCG), outils informatiques extrêmement lourds, sont capables de simuler les grands traits de la réponse du système climatique terrestre en fonction d'un scénario donné, comme par exemple celui consistant à doubler le gaz carbonique $\left(\mathrm{CO}_{2}\right)$ dans l'atmosphère. Malheureusement, ils ne peuvent être utilisés tels quels dans le cadre d'études d'impact sur une zone aussi petite que les Alpes françaises, essentiellement à cause de la taille de leur maille et de leur façon de représenter le relief.

Le but du travail présenté ici est de développer et d'utiliser des outils permettant d'estimer les conséquences d'un changement du climat sur l'enneigement des Alpes françaises. L'étude se concentrera tout d'abord sur la modélisation de la climatologie nivale des Alpes à partir des données météorologiques observées et à des tests de sensibilité. Ensuite, pour prendre en compte de façon plus satisfaisante les scénarios des modèles de circulation générale, une méthode de régionalisation adaptée sera développée, afin de permettre le fonctionnement des outils de modélisation du manteau neigeux à partir des sorties d'un modèle de circulation générale. Ainsi, l'impact de deux scénarios de « doublement du $\mathrm{CO}_{2}$ » sur l'enneigement des Alpes pourra être discuté.
\end{abstract}

\section{IINTÉRÊT DE LA MODÉLISATION NU- MÉRIQUE}

L'examen des mesures de l'enneigement souligne la forte variabilité du paramètre neige (à la fois dans le temps et l'espace). Mais ces données ne peuvent pas être utiles dans le cadre d'une étude d'impact des changements du climat sur l'enneigement à l'échelle des Alpes parce que la représentativité spatiale des données reste faible et qu'il existe dans la physique de la neige de nombreux effets de seuil (changements de phase par exemple) qui font qu'il est souvent difficile de relier la hauteur de neige à un instant donné avec les paramètres météorologiques classiques comme la température et les précipitations. C'est pour cela que l'on s'est tourné vers une approche numérique permettant la modélisation de l'ensemble des phénomènes physiques. Cette approche permet, en outre, de faire facilement des tests de sensibilité aux données météorologiques d'entrée.

Deux outils numériques ont été utilisés. Le premier est un modèle unidimensionnel d'évolution du manteau, appelé CROCUS [1], [2]. Ce dernier simule l'évolution du manteau neigeux en fonction des conditions météorologiques au-dessus de la surface. Pour obtenir des simulations de l'enneigement à l'échelle des Alpes, on a associé au système d'analyse des données météorologiques SAFRAN [3]. Pour cela, les Alpes françaises ont été divisées en 23 massifs (d'environ $1000 \mathrm{~km}^{2}$ ), considérés comme homogènes d'un point de vue météorologique. SAFRAN est capable de fournir les paramètres d'entrée de CROCUS (rayonnement infrarouge et solaire incident, température, humidité, et force du vent au-dessus de la surface, précipitations) sur l'ensemble de ces massifs à différentes altitudes. SAFRAN utilise pour cela l'ensemble des informations météorologiques disponibles (observations et résultats de modèles météorologiques).

La crédibilité d'une étude de sensibilité climatique réalisée avec SAFRAN et CROCUS dépend en grande partie de sa capacité à reproduire la climatologie nivale actuelle, ainsi que sa variabilité. C'est pour cela que ces deux outils ont été validés en comparant hauteurs de neige simulées et mesurées sur 37 sites des Alpes françaises sur une période de dix ans. L'erreur sur ce paramètre est généralement inférieure à $30 \mathrm{~cm}$ (fig. 1). Ceci montre la bonne qualité de la simulation, puisqu'une erreur commise dans l'analyse des paramètres météorologiques est susceptible d'avoir un effet sur les simulations de hauteur de neige pendant le reste de la saison. Les autres sources d'erreur sont le transport de neige par le vent, ou des effets locaux d'échelle inférieure au massif (vallées encaissées), qui ne sont pas pris en compte. Mises à part les quelques restrictions évoquées ci-dessus, la reproduction de l'enneigement décadaire moyen sur 10 ans est très satisfaisante et montre la capacité de SAFRAN et CROCUS à reproduire de manière très satisfaisante la climatologie nivale actuelle. 


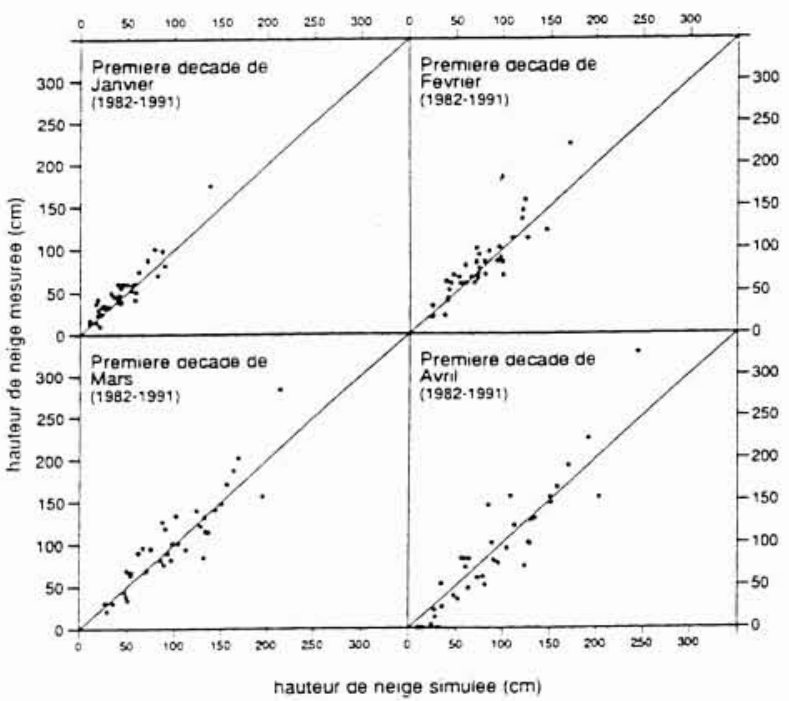

1. Hauteurs décadaires moyennes de neige mesurées et simulées pour les 37 sites durant les premières décades de janvier, février, mars et avril.

\section{II $\square$ TESTS SIMPLES DE SENSIBILITÉ DE L'ENNEIGEMENT}

Une fois la bonne qualité de la chaîne SAFRAN/CROCUS vérifiée, il est possible de l'utiliser pour des études d'impact. Diverses expériences ont été effectuées [4]. Elles ont consisté en la modification d'un ou plusieurs paramètres météorologiques d'entrée du modèle CROCUS, comme la température de l'air, la phase et la quantité des précipitations. On a également utilisé un scénario climatique correspondant à un doublement du contenu en gaz carbonique $\left(\mathrm{CO}_{2}\right)$ dans l'atmosphère. Une analyse des variations des paramètres météorologiques sur les Alpes données par le modèle de circulation générale EMERAUDE [5] a permis d'élaborer un scénario. Bien sûr, ce type d'approche est loin d'être satisfaisant vu la taille de la maille des modèles de circulation générale, mais l'objectif visé était surtout d'obtenir des perturbations cohérentes entre elles, des différents paramètres. Les perturbations des variables météorologiques correspondant au scénario «doublement du $\mathrm{CO}_{2}$ " ont été les suivantes : rayonnement infrarouge incident : $+3 \%$, température de l'air : $+1,8^{\circ} \mathrm{C}$ transformation de la neige en pluie lorsque la nouvelle température de l'air dépasse $1.5^{\circ} \mathrm{C}$.

Sans entrer dans le détail des résultats, on peut dire que deux paramètres (ou groupes de paramètres) sont importants pour le manteau neigeux. Le premier est la température de l'air (et le rayonnement infrarouge, qui lui est lié). Une limite se dessine entre 2000 et $2500 \mathrm{~m}$. Au-dessus, l'effet d'une augmentation de la température, est faible et limité au printemps (accélération de la fonte). Au-dessous, les effets se font sentir même pendant la saison hivernale. Le deuxième paramètre important est constitué par l'évolution des quantités de précipitations. Son effet est a priori plus facile à appréhender. Par exemple, si on augmente les quantités de précipitations, les calculs montrent qu'on retrouve à peu près la même variation sur l'épaisseur de neige. Par contre la variation est très faible pour ce qui concerne la durée de l'enneigement. En effet, la neige tombée en surplus disparaît en fin de saison, au moment où la température est élevée et la fonte rapide. On obtient des résultats symétriques si on diminue les quantités de précipitations.

L'impact du scénario climatique décrit ci-dessus est très important, que ce soit pour l'épaisseur de neige ou la durée de l'enneigement. Cette dernière, à $1500 \mathrm{~m}$, diminue de 30 à 40 jours par an en moyenne, passant par exemple de 170 à 130 jours par an dans la région du Mont-Blanc et de 100 à moins de 60 jours pour les massifs situés à l'extrême sud. La figure 2 décrit l'évolution de la hauteur maximale de neige (en moyenne décadaire sur dix ans) en fonction de la durée moyenne de l'enneigement telles qu'elles ont été simulées par CROCUS dans la simulation de référence et pour diverses expériences de sensibilité (massif du MontBlanc, à 1500 et à $3000 \mathrm{~m}$ ). On remarque bien les effets différenciés de la température et des précipitations. En particulier, il semble improbable qu'une augmentation des précipitations puisse compenser l'effet d'un éventuel réchauffement à basse altitude.

\section{III ÉLABORATION D'UNE PROCÉDURE DE RÉGIONALISATION DES RÉSUL- TATS DE MODÈLES DE CIRCULATION GÉNÉRALE (MCG)}

\subsection{Présentation de la méthode utilisée}

Le principal défaut des scénarios décrits dans le paragraphe précédent tient au fait que les perturbations des paramètres météorologiques sont identiques quels que soient la période, le massif ou l'altitude. On ne prend pas en compte, par exemple, un déplacement des centres d'actions météorologiques, qui modifierait les types de temps affectant la région des Alpes. Or, il est possible que ce genre de modification ait une influence comparable à celle d'une simple variation de la température. Pour améliorer la prise en compte de l'information des MCG, une méthode de régionalisation adaptée aux outils SAFRAN et CROCUS a été développée. L'intérêt de ces méthodes est qu'elles permettent, à partir de l'information à grande échelle fournie par le MCG d'estimer les paramètres nécessaires à l'échelle souhaitée.

On a utilisé une méthode de régionalisation inspirée des techniques de prévision météorologique par recherche de situations analogues. Celle-ci consiste à associer dans un premier temps à un état de l'atmosphère simulé par un modèle de circulation générale pendant une période donnée (une journée) un état réel de l'atmosphère analogue (c'està-dire le plus ressemblant possible) sélectionné dans un fichier de référence. Ce sont les champs en altitude (700 ou $500 \mathrm{hPa}$ ) qui seront utilisés pour déterminer le degré de similarité des deux journées. Du point de vue pratique, cette méthode peut être décomposée en trois étapes (fig. 3) :

Etape 1: Chaque journée de simulation du modèle de circulation générale, est associée à une journée réelle analogue (c'est-à-dire la plus ressemblante du point de vue des champs en altitude, celle-ci étant extraite d'un fichier de référence à définir). 

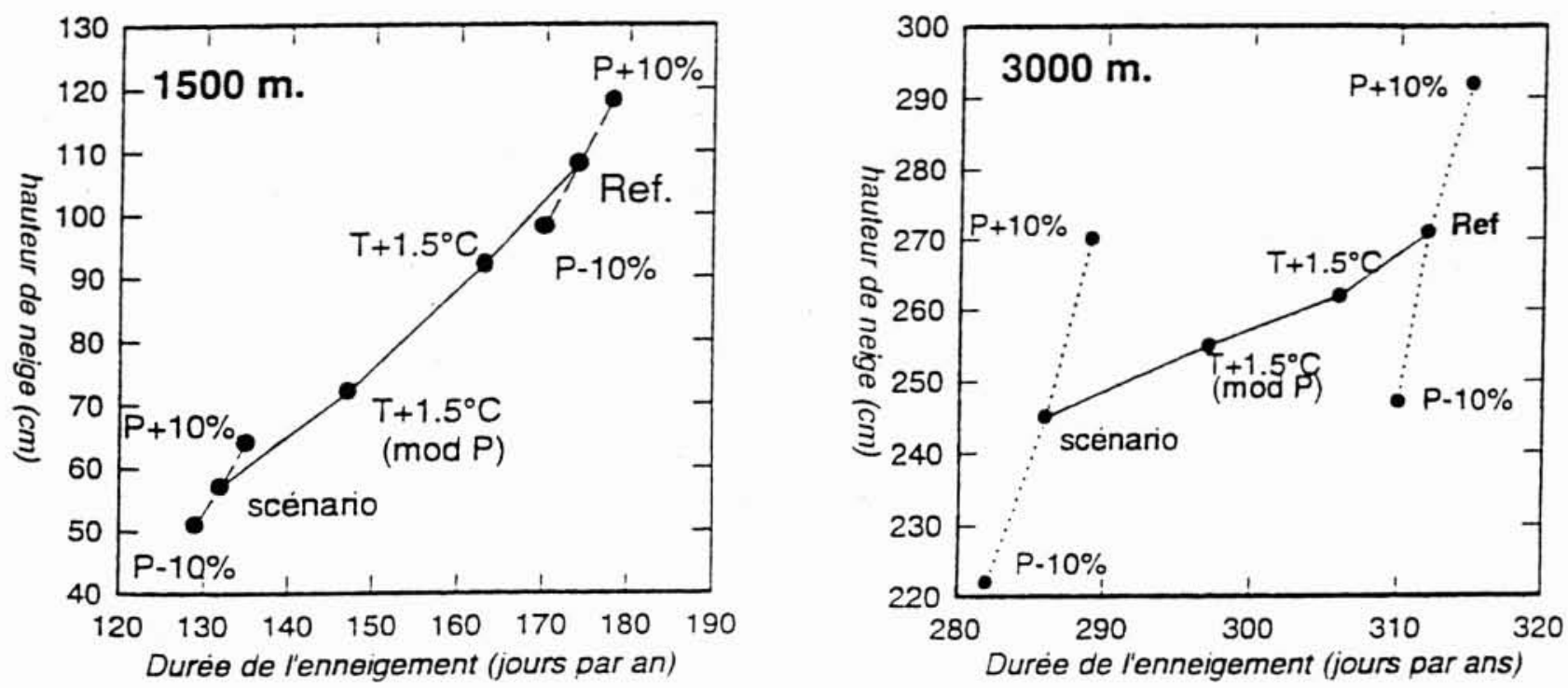

2. Evolution de la durée moyenne d'enneigement et de la hauteur moyenne de neige pendant la première décade de mars en fonction de diverses hypothèses pour le massif du Mont-Blanc à $1500 \mathrm{~m}$ (à gauche) et à $3000 \mathrm{~m}$ (à droite). Références : conditions actuelles, $\mathrm{T}+1,5^{\circ} \mathrm{C}$ : augmentation de la température de l'air de $1,5^{\circ} \mathrm{C}, \mathrm{T}+1,5^{\circ} \mathrm{C},(\mathrm{mod}$ P) : idem plus transformation de la neige en pluie si la nouvelle température de l'air dépasse $1,5^{\circ} \mathrm{C}$. Scénario : scénario complet, $P+10 \% P-10 \%$ : variation des quantités de précipitations de $10 \%$.

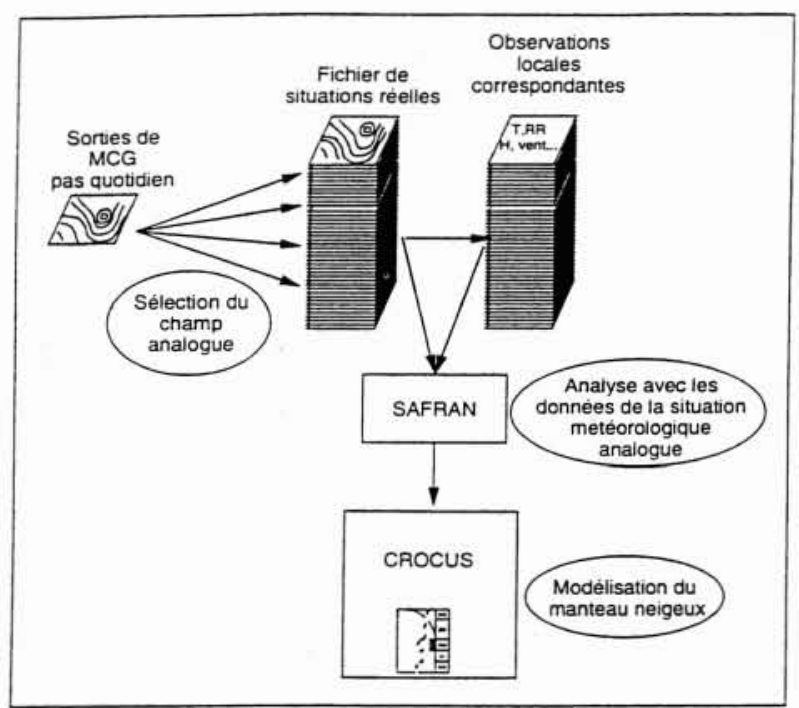

3. Principe général de la procédure de régionalisation.

Etape 2 : SAFRAN analyse les conditions météorologiques sur les sites choisis. Ses données d'entrée sont constituées par l'ensemble des données disponibles de la journée réelle analogue (champs en altitude et observations en surface).

Etape 3 : CROCUS simule l'enneigement à partir des données fournies par SAFRAN.

Si les étapes 2 et 3 ne posent pas de problèmes puisque SAFRAN et CROCUS sont des outils validés, il n'en est pas de même pour l'étape 1 pour laquelle une procédure a été développée et testée.

\subsection{Tests sur les données réelles}

Le fichier de référence est constitué par les analyses météorologiques du modèle du CEPMMT (Centre européen pour les prévisions météorologiques à moyen terme). Il contient les analyses du géopotentiel, de la température et de l'humidité à 700 et $500 \mathrm{hPa}$, du $1^{\text {er }}$ août 1981 au 31 juillet 1991, à 00 et 12 UTC. Concernant la méthode de sélection, c'est une méthode de type "plus proche voisin » qui est utilisée. Le critère de sélection, ou distance, est la somme des distances euclidiennes sur les valeurs des champs de géopotentiel et de température sur les points de grille à 700 et $500 \mathrm{hPa}$ au-dessus des Alpes. Les distances sur les différents paramètres sont pondérées pour tenir compte des différences dans les distributions statistiques.

Le critère a été sélectionné parmi de nombreux critères basés sur d'autres variables (humidité, gradient de géopotentiel, advection de température, tourbillon de géopotentiel...). Les performances relatives de chaque critère ont été testées sur le fichier de dix années d'analyses. Le test a été fait de la manière suivante : à chaque journée du fichier, on associe la journée la plus ressemblante du point de vue des critères déjà évoqués. Pour que les situations météorologiques soient indépendantes, on s'interdit de sélectionner de journées voisines de la journée de référence comme analogue. De plus, on souhaite utiliser l'ensemble des données analysées par SAFRAN pendant les journées analogues. Cela impose donc des contraintes supplémentaires, car il faut que les termes de rayonnement (solaire en particulier) soient comparables. Ainsi, les analogues devront être situés dans une fenêtre glissante de $+/-15$ jours autour de la date de la situation de référence. L'analogue de la journée sera sélectionné parmi un peu moins de 300 journées possibles ( 30 jours $\times 10$ ans, moins les journées voisines de la journée de référence). 


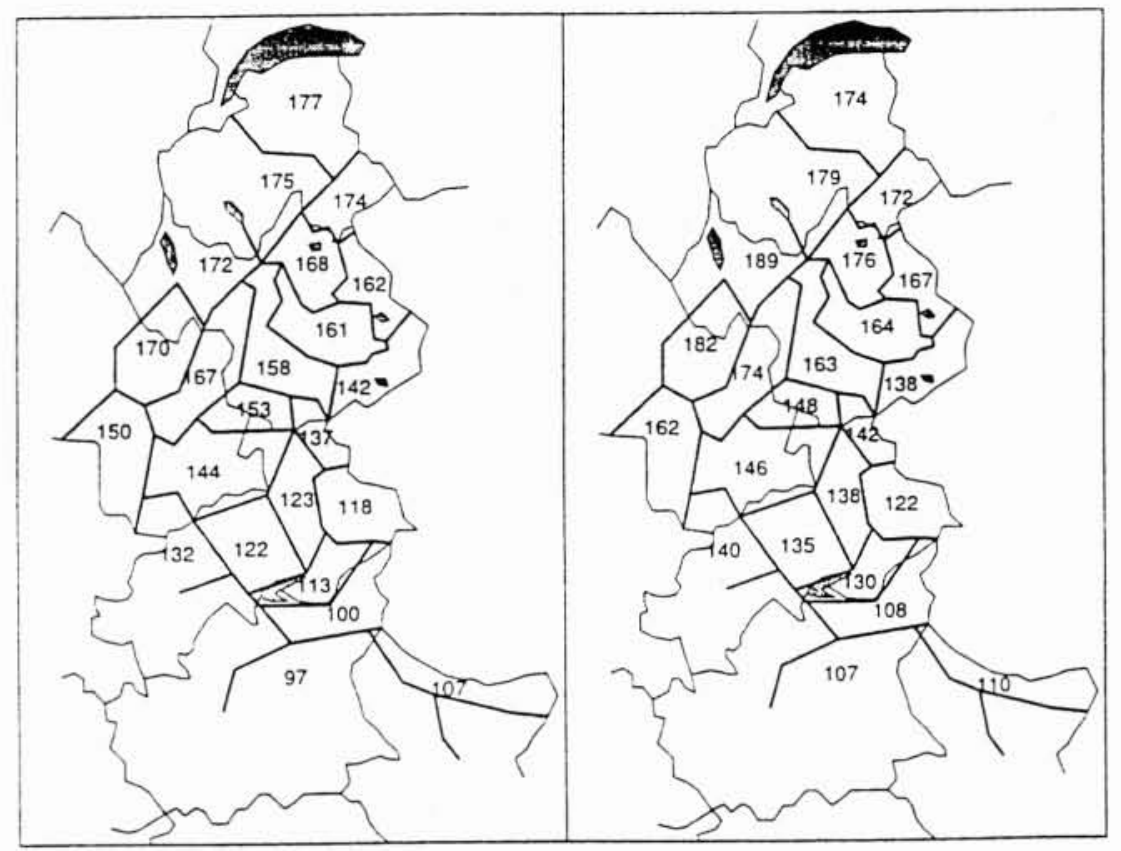

4. Durée moyenne de l'enneigement à $1500 \mathrm{~m}$ (jours/an) pour les 23 massifs. La climatologie SAFRAN/CROCUS (à gauche) est comparée à la reconstitution effectuée avec les journées analogues (à droite).

A la chronologie originale de 3650 journées, une nouvelle chronologie est associée. Le test de la série analogue a été fait sur les trois paramètres les plus importants : les précipitations, la température de l'air et la hauteur de neige simulée par CROCUS. Pour les deux premiers, on s'est intéressé aux résultats obtenus sur deux stations météorologiques, Bourg-Saint-Maurice $(868 \mathrm{~m})$, dans les Alpes du nord et Embrun $(876 \mathrm{~m})$, dans les Alpes du sud. Les vérifications sur les hauteurs de neige ont été effectuées à partir des résultats de la chaîne SAFRAN/CROCUS alimentée par les données des journées analogues. La figure 4 permet de comparer, pour la durée de l'enneigement à $1500 \mathrm{~m}$ la climatologie de référence SAFRAN/CROCUS à celle obtenue grâce aux journées analogues. Ces résultats tout à fait satisfaisants prouvent que la méthode de sélection des analogues est adaptée pour retrouver les principales caractéristiques de la climatologie nivale des Alpes françaises.

\section{APPLICATION A DES SORTIES DE MODÈLES DE CIRCULATION GÉNÉ- RALE}

\subsection{Les simulations utilisées}

Trois simulations réalisées avec le modèle ARPEGE [6] en troncature T42 ont été utilisées. La première appelée "col» est une simulation de référence représentant le climat actuel. Les deux autres expériences appelées «co2» et « uk2 2 sont deux expériences de type « doublement de $\mathrm{CO}_{2}$ " [7]. Les variations des températures de la mer introduites comme condition aux limites pour les simulations ARPEGE proviennent de deux simulations transitoires avec océan global réalisées au Max Planck Institute für Meteorologie (MPI, pour co2) et au Hadley Centre (HC pour uk2). Dans les deux cas, ces variations ont été calculées au moment du doublement du $\mathrm{CO}_{2}$, les différences entre les deux expériences proviennent des différences dans les formulations des deux modèles. Ainsi, l'augmentation moyenne des températures de surface de la mer est plus forte pour le modèle du Hadley Centre que pour celui du Max Planck Institute $\left(1,14^{\circ}\right.$ au lieu de $\left.0,96^{\circ}\right)$.

\subsection{Reconstitution du climat actuel}

La méthode utilisant une sélection de journées analogues basée sur le géopotentiel et la température ne peut que répercuter les biais éventuels existants pour ces paramètres dans la simulation du MCG. La température est évidemment une variable particulièrement sensible pour la simulation du manteau neigeux. Or les températures de la simulation col sont légèrement supérieures à la climatologie du CEPMMT pendant la période hivernale, surtout à $700 \mathrm{hPa}$. De novembre à mars à $500 \mathrm{hPa}$, la moyenne des températures pour l'expérience col est égale à celle des analyses du CEPMMT. Mais une légère sous-estimation en automne masque une surestimation au printemps qui peut avoir des conséquences importantes sur la fonte. A $700 \mathrm{hPa}$ la surestimation est de $0,8^{\circ} \mathrm{C}$, les différences étant particulièrement marquées en fin d'hiver.

L'originalité de la méthode de régionalisation proposée est de tenir compte du type de situation au jour le jour. Or, une des variables importantes pour le caractériser est la direction générale du flux. L'examen de roses du vent géostrophique, tracées pour un point situé au centre des Alpes françaises à $700 \mathrm{hPa}$ montre que celles-ci sont très différentes de la climatologie : les secteurs sud-ouest, ouest et nord-ouest, regroupant $85 \%$ des journées, sont nettement prédominants (50\% dans la climatologie). De plus, la circulation étant plus marquée que dans la climatologie. il faut donc s'attendre à une surestimation des précipitations par la méthode des analogues (fréquence plus importante des passages perturbés). Les reconstitutions de l'enneigement faites à partir des journées réelles analogues (fig. 5, en haut, pour la durée de l'enneigement à $1500 \mathrm{~m}$ ) ont été comparées à la climatologie SAFRAN/CROCUS. La durée de l'enneigement est restituée de manière satis- 


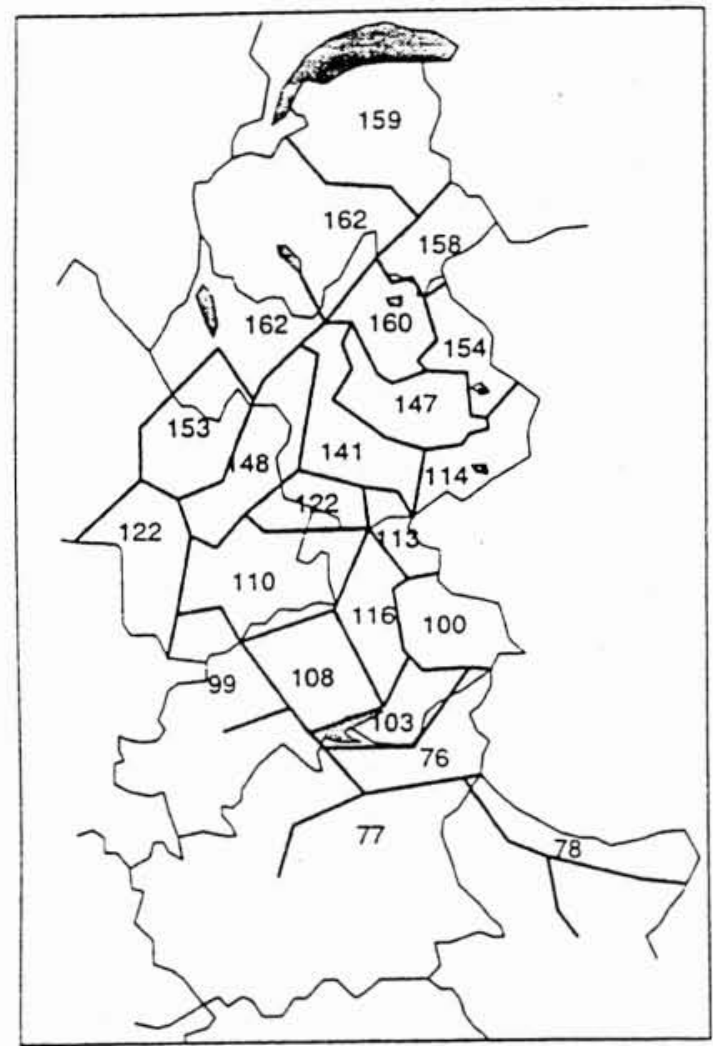

\section{$\leftarrow$ expérience co1}

$K$ expérience $\operatorname{co} 2$ (MPI)

$\downarrow$ expérience uk2 (HC)

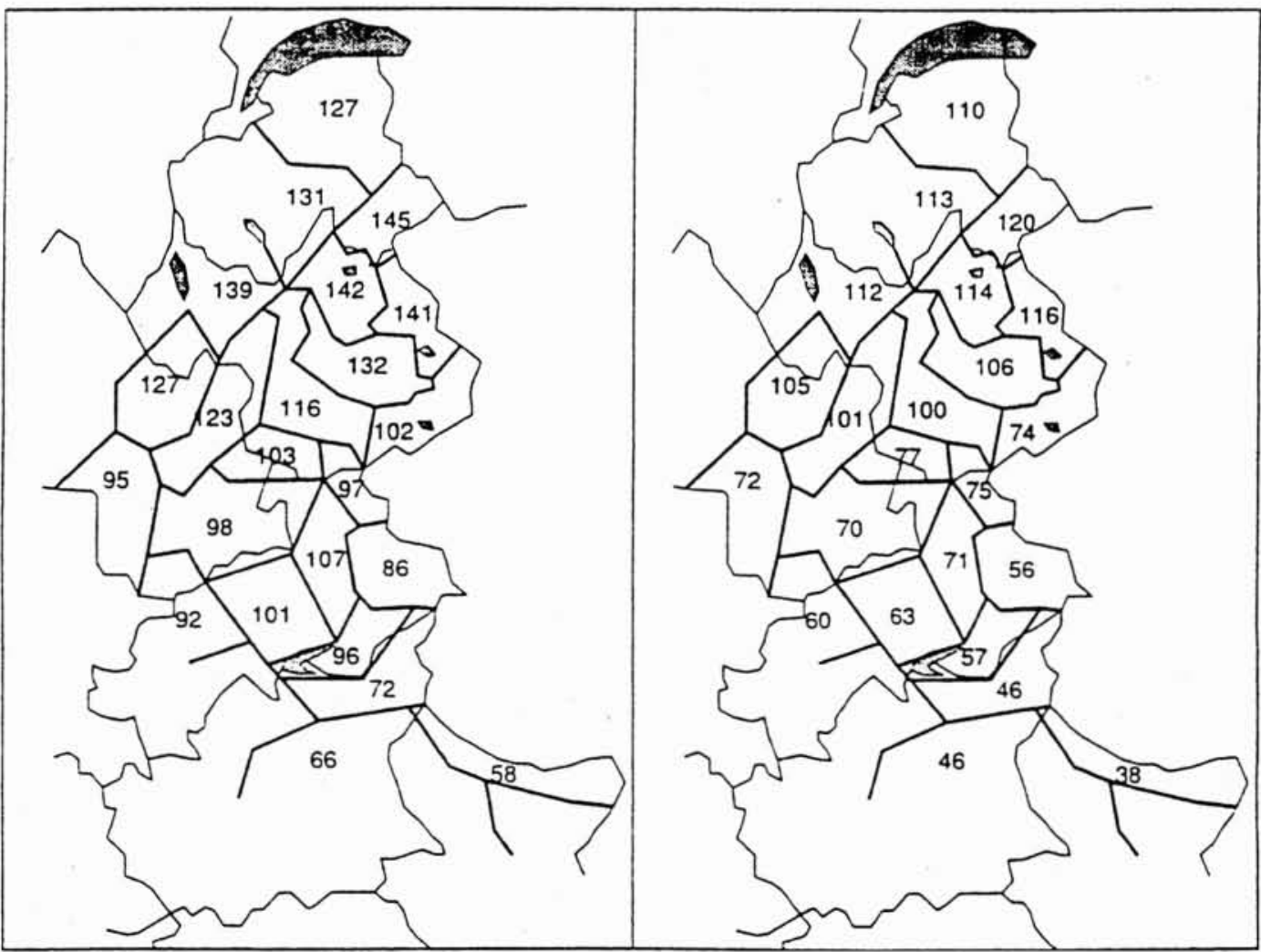

5. Durée moyenne de l'enneigement à $1500 \mathrm{~m}$ (en jours/an) pour les 23 massifs. Les résultats de l'expérience co1 (en haut à gauche) sont comparés à ceux des expériences $\operatorname{co2}$ (en bas à gauche) et uk2 (en bas à droite) : reconstitution par la méthode des analogues. 
faisante mais est sous-estimée, essentiellement à cause de la surestimation de la température.

\subsection{Impact d'un doublement du gaz carbonique sur l'en- neigement}

Les deux scénarios de doublement du $\mathrm{CO}_{2}$ se traduisent par un réchauffement des températures à 700 et $500 \mathrm{hPa}$. Pour ce qui concerne l'expérience co2 (MPI), le réchauffement est minimal au printemps (mars-avril), l'augmentation de la fonte devrait être limitée pendant cette période (l'augmentation de la température de novembre à mars est de $1,1^{\circ} \mathrm{C}$ à $700 \mathrm{hPa}$ et de $1.8^{\circ} \mathrm{C}$ à $500 \mathrm{hPa}$, toute l'année elle est respectivement de $1,3^{\circ} \mathrm{C}$ et $1,9^{\circ} \mathrm{C}$ ). Pour l'expérience uk2 (HC), le réchauffement est plus intense (le minimum du printemps est moins marqué). L'augmentation de la température de novembre à mars est de $2,0^{\circ} \mathrm{C}$ à $700 \mathrm{hPa}$ et de $2.7^{\circ} \mathrm{C}$ à $500 \mathrm{hPa}$, toute l'année elle est respectivement de $2,6^{\circ} \mathrm{C}$ et $3,0^{\circ} \mathrm{C}$.

Les effets sont très différents sur les précipitations. Dans le cas de l'expérience col, on assiste à une augmentation des précipitations hivernales totales, ce qui limite l'effet de l'augmentation de la température. Ainsi, à $3000 \mathrm{~m}$, les chutes de neige augmentent légèrement. Pour l'expérience $\mathrm{uk} 2$, les précipitations totales et les chutes de neige sont en baisse à tous les niveaux.

A $1500 \mathrm{~m}$. les variations de la durée de l'enneigement (fig. 5) sont surtout marquées par l'augmentation de la température. La diminution de la durée de l'enneigement est donc générale. Pour l'expérience co2, la diminution la plus marquée se situe dans les Préalpes du nord (Bauges, Aravis, Chablais : 30 jours ou plus). La diminution la plus faible se situe dans les Alpes du sud (Dévoluy, Champsaur, Parpaillon, Ubaye : moins de 10 jours. C'est une conséquence d'une augmentation plus faible de la température dans le sud $\left(0,7^{\circ} \mathrm{C}\right)$ que dans le nord $\left(1,0^{\circ} \mathrm{C}\right)$ et de l'augmentation des chutes de neige dans les Préalpes du sud, les deux effets étant de même sens. Pour l'expérience uk2, la diminution est très uniforme (40 jours par an environ).

A $3000 \mathrm{~m}$, la diminution de la durée de l'enneigement est limitée à quelques jours par an pour $\mathrm{co} 2$ (moins de 10 jours en général), les différences régionales trouvées à $1500 \mathrm{~m}$ ne sont pas visibles car ce paramètre est peu sensible à haute altitude aux variations des précipitations. Pour l'expérience uk2, la forte augmentation de la température (conjuguée à la forte diminution des chutes de neige) entraîne des baisses importantes de la durée de l'enneigement ( -15 à -40 jours). Les variations les plus importantes sont localisées dans le sud, le massif le plus touché étant le Mercantour.

\section{$\mathrm{V} \square$ CONCLUSION}

La modélisation s'est avérée être un bon moyen de reconstituer la climatologie nivale des Alpes françaises. Grâce à une validation effectuée sur une période de dix ans, SAFRAN et CROCUS ont pu être mis en œuvre pour reconstituer des données météorologiques et d'enneigement homogènes sur l'ensemble de la zone. Grâce au développement d'une méthode de régionalisation adaptée. ces outils ont pu en outre être utilisés à partir de sorties de modèles de circulation générale. La méthode développée ici, basée sur la recherche de journées réelles analogues s'est révélée être efficace et pourrait être utilisée sur d'autres régions.
Deux approches ont été employées pour estimer l'impact d'un changement du climat sur l'enneigement des Alpes françaises.

- La première a consisté à perturber les paramètres météorologiques à partir de la situation de référence et à observer les conséquences sur le manteau neigeux. Cette méthode présente l'avantage de fournir un état de référence aussi proche que possible de la réalité, mais le scénario climatique ne peut être que très simple.

- La deuxième a consisté à utiliser une méthode de régionalisation des modèles de circulation générale. Cette solution est plus séduisante a priori puisqu'elle permet d'évaluer l'impact sur le manteau neigeux de tout scénario climatique simulé par ce type de modèle. En revanche, l'état de référence correspondant au climat actuel est reconstitué avec une précision moindre que dans la première méthode.

Les deux méthodes permettent de quantifier la réponse de l'enneigement à différents scénarios climatiques. Elles ont mis en évidence la forte sensibilité des altitudes moyennes, ainsi que la différence de réponse entre la partie nord et la partie sud des Alpes françaises. La méthode de régionalisation pourra être utilisée à l'avenir pour étudier tout scénario climatique fourni par un modèle de circulation générale. Evidemment, l'étude d'impact ne doit pas s'arrêter uniquement aux épaisseurs de neige et durée de l'enneigement, l'aspect impact sur les ressources en eau est également très important. Mais la comparaison des impacts des deux scénarios de doublement de $\mathrm{CO}_{2}$ montre bien les efforts à mener dans les domaines de la modélisation de la circulation générale et de la régionalisation pour aboutir à des scénarios climatiques régionaux fiables.

\section{Bibliographie}

[1] Brun E., martin E., Simon V., Gendre C., Coléou C. (1989). - An energy and mass model of snow cover suitable for operational avalanche forecasting. J. of Glaciol., Vol. 35. $\mathrm{N}^{\circ} 121$, p. 333-342.

12] Brun E., David P.. Sudul. M.. Brunot G. (1992). - A numerical model to simulate snowcover stratigraphy for operational avalanche forecasting, J. of Glaciol., Vol. $38, \mathrm{~N}^{\circ} 128$. p. 13-22.

[3] Durand Y., Brun E., Mérindol. L.. Guyomarc'H G., LeSAFRE B., MARTIN E. (1993), - A meteorological estimation of relevant parameters for snow models. Annals of Glaciol., Vol. 18, p. 65-71.

14] Martin E., BRUn E., Durand Y. (1994). - Sensitivity of the French Alps snow cover to the variation of climatic variables. Annales Geophysicae, 12, 469-477.

[5] Mahfouf J.-F., Royer J.-F., CARIOll.e D. (1992). - A $2 * \mathrm{CO}_{2}$ experiment with prescribed changes in sea surface temperature. Proceedings of the central symposium of the "International space year" conference, Munich, 30 mars4 avril 1992 (ESA SP-341, July 1992), p. 151-155.

[6] Déqué M., Dreveton C., Braun A., Cariolle D. (1994). - The ARPEGE/IFS atmosphere model : a contribution to the French community climate modelling. Climate Dynamics. 10, p. 249-266.

17] Timbal B., Mahfouf J.-F. (1993). - Analyses d'expériences de sensibilité au doublement du $\mathrm{CO}_{2}$ avec le modèle AR PEGE : comparaison avec les résultats d'EMERAUDE. Note de travail du groupe de météorologie à grande échelle et climat $n^{\circ} 29$ 